\title{
SOME RADICAL CONSEQUENCES OF GEACH'S LOGICAL THEORIES
}

\author{
By James CAin
}

DETER Geach's views of relative identity, together with his account of proper names and quantifiers, ${ }^{1}$ while presenting what I believe is an inwardly coherent and consistent account, presents us with radical consequences regarding what arguments are to be accepted as valid. For example, consider the argument:

All men are mortal

Fido is a man

Thus, Fido is mortal

in which 'Fido' names a dog. While this would normally be thought to be a valid argument with a false premise, we shall see that on Geach's theories it turns out to be invalid. In fact for every form of general categorical syllogism we can produce an argument of that form which on Geach's theories turns out invalid.

${ }^{1}$ I will only be concerned with the theory of restricted quantifiers worked out in the main body of Reference and Generality, third edition (Ithaca: Cornell University Press, 1980), not with either his theory of unrestricted quantifiers or the method of interpreting restricted quantifiers mentioned in the appendix to Reference and Generality. 
Let us see how this situation arises. We first look briefly at Geach's semantics. According to Geach every proper name is associated with a criterion of identity which could be expressed using a substantival term; e.g., 'Fido' is, let's say, associated with the criterion of identity given by 'same dog': this can be expressed by saying that 'Fido' is a name for a dog (we may go on to say that it is a name of a dog if it is non-empty; Reference and Generality p. 70). Restricted quantification is in turn explained in terms of proper names. Geach says:

' $F$ (some $A$ )' is true iff ' $F(a)$ ' is true for some interpretation of ' $a$ ' as a name of and for an $A$;

' $F($ any $A)$ ' is true iff ' $F(a)$ ' is true for any interpretation of ' $a$ ' as a name of and for an $A$.

(These schematic conditions are of course to be applied to actual concrete examples by replacing the letter ' $A$ ' with some actual general term both within and outside the quotes.) (Reference and Generality p. 206)

Since we will eventually also consider phrases of the form 'No $A$ ', let us supplement Geach's definition for restricted quantifiers with:

' $F($ no $A)$ ' is true iff ' $F(a)$ ' is true for no interpretation of ' $a$ ' as a name of and for an $A$.

Now we turn to Geach's views on identity. According to Geach's views we may have cases in which $a$ and $b$ are the same $A$ yet we may be able to say conflicting things about them. One example involves heralds; Geach introduces us to heralds as follows:

In England the members of the Heralds' College have a range of special names, such as 'Garter' 'Rouge Dragon', 'Portcullis'. These are grammatically, and I think logically, proper names. Each of these is a name for a herald; by repeated use of it we have in mind not one and the same man, but one and the same herald - the filler (whoever he may be) of one and the same heraldic office. ('Existential or Particular Quantifier' in Weingartner and Morscher (eds.) Ontology and Logic, Dunker and Humblot, 1979, p. 146)

In the typical case in which just one man holds a given office in the Heralds' College we will be able to say, according to Geach, that the herald is a man. However, at different times one herald may be different men, as in the following example of Geach:

At a given point of time the man Hilary Handel is a herald, in fact he is (the same herald as) the herald Sable Basilisk; conversely, at the same point of time the herald Sable Basilisk is a man, in fact he is (the same man as) the man Hilary Handel ... one and the same herald, say Sable Basilisk, may at different times be (be the same man as) different men. (Reference and Generality p. 217)

Even though, let's suppose, Sable Basilisk and Hilary Handel are now the same man, conflicting predicables might apply to them, e.g., ' - has existed for more than fifty years' might apply to Sable Basilisk, but not to Hilary Handel. 
Now consider the argument:

All men are mortal.

Sable Basilisk is a man.

Thus, Sable Basilisk is mortal. ${ }^{2}$

In the light of the fact that heraldic posts may be held by a succession of men, and therefore a herald might exist forever (as far as logic is concerned), it is clear that the conclusion does not follow from the premises. The argument turns out to be invalid. (At this point we need to see that the first premise comes out true, according to Geach's semantics, as long as ' $a$ is mortal' is true for any possible reading of ' $a$ ' as a name of and for a man: Sable Basilisk might exist forever and the first premise still hold since 'Sable Basilisk' is not a name for a man.) We now also see why argument (1) must also be treated as invalid according to Geach's theory: it is logically on a par with argument (2).

This sort of consideration also shows the following arguments to be invalid (imagine the case in which no herald ever ceases to exist, yet all heralds are men):

All men are mortal.

All heralds are men.

Thus, all heralds are mortal.

All men are mortal.

Some herald is a man.

Thus, some herald is mortal.

And these would typically be taken to be valid syllogisms. Using our imagined example in which heralds never cease to exist, we could find arguments in which the premises come out true and the conclusion false corresponding to each form of general categorical syllogism in the first three figures traditionally regarded as valid. These arguments are merely slight variations upon our last two examples, so I need not list them. (In each case either 'mortal' or 'immortal' corresponds to the term which does not ever appear in subject position. 'Man' and 'herald' correspond to the other terms.)

Even Geach, up through his third edition of Reference and Generality, seems to have been unaware of the shake-up that his theories cause in what arguments we must recognize as valid. To give just one example, Geach takes the following to be a valid argument form: 'Socrates in an $A$; any $A$ is a donkey; ergo Socrates is a donkey'. (Reference and Generality p. 143) Surely the validity of this form stands or falls with the validity of:

\footnotetext{
${ }^{2}$ If one feels uncomfortable using the predicable 'is mortal' when speaking of heralds, one may substitute the predicable 'will have ceased to exist'; or we may stipulate that in this paper 'is mortal' shall mean 'will have ceased to exist'.
} 
$a$ is an $A$.

All $A$ are $F$.

Thus, $a$ is an $F$.

But we have already shown arguments (1) and (2) to be invalid in Geach's system, and they are instances of the above with the order of the premises switched.

Consider further Geach's views on identity. Geach gives the following example which would confirm Wiggins' thesis $R$ :

A parliament intent on economy might decree that in the national emergency Norroy shall be the same man as Clarencieux; in that case 'Sir Hilary Handel', a name for a man, might be a name shared by two different heralds, Norroy and Clarencieux, and yet be a proper name of a man, since it names but one man and ex hypothesi each of these heralds will be the same man as Sir Hilary Handel. ('Existential or Particular Quantifier' p. 148)

Note that we could not, in this case, say that Sir Hilary was a herald. For it would be absurd to say that he was the same herald as just one of the two. Nor would he be the same herald as both, or by the transitivity and symmetry of 'is the same herald as' Clarencieux and Norroy end up being the same herald - but the example stipulates that they are not. The only alternative left if Handel is to be a herald is that he be a different herald from Clarencieux and Norroy, but this gives the unwanted result that we have three heralds. We must conclude that Handel is not a herald.

Let us extend this last example by imagining that the human race is all but destroyed, so that Handel is the only human left, and Norroy and Clarencieux are the only heralds left. Since Handel is not a herald we have:

No men are heralds

coming out true and yet its converse:

No heralds are men

coming out false since both Clarencieux and Norroy are men. Yet this pair of propositions would normally be regarded as an instance of an equivalent pair of schemata:

No $A$ are $B$

and,

No $B$ are $A$.

Consider also the argument:

No humans are heralds.

Some heralds are human.

Thus, some humans are not human.

Our example would make the premises come out true and the conclusion false, though this would typically be regarded as a valid 
syllogism of the fourth figure, being schematized:

No $A$ are $B$.

Some $B$ are $C$.

Thus, some $C$ are not $A$.

In fact, using our example, we may provide invalid arguments exemplifying each form of argument generally recognized as valid in the fourth figure. So there are counterexamples to each form of general categorical syllogism generally recognized as valid.

This last example, by the way, provides a counterexample to the following, which Geach offers as a valid schema ('Names and Identity' in Guttenplan (ed.) Mind and Language, Oxford 1975, p. 140):

Every $A$ is a $B$ or is a $C$.

Every $B$ is $P$; every $C$ is $P$.

Thus, every $A$ is $P$.

Consider this interpretation of the schema:

Every herald is a man or is a man.

Every man is not a herald; every man is not a herald. Thus, every herald is not a herald.

Our example makes the premises true and the conclusion false.

Geach's theory not only drives us to treat as invalid arguments that would normally be treated as valid, but also to treat as valid arguments that would normally be treated as invalid. Consider the following argument in which 'Socrates' functions as a name of and for a man:

All men are mortal.

Thus, Socrates is mortal.

This argument is usually regarded as an enthymeme. On Geach's theory it should be regarded as strictly valid. Since, on Geach's view, it is part of the sense of 'Socrates' that it is a name for a man (Reference and Generality pp. 70-1), we are justified in concluding that Socrates is mortal, if we are given that all men are mortal. This is shown as follows: the premise comes out true iff ' $a$ is mortal' is true under any possible reading of ' $a$ ' as a name of and for a man; 'Socrates' presents us with such a reading of ' $a$ ' (Geach holds that empty names are inadmissible in logic - so we need not worry about 'Socrates' not being a name of a man); thus with 'Socrates' replacing ' $a$ ' we get a truth whenever the premise is true. So the conclusion follows from the premise and the argument is valid.

Geach does provide an example in which we get a valid argument where it would normally be thought that we have an enthymeme (Reference and Generality p. 109). He allows that from the premise 'no man has admired any pig' we may infer 'the man $a$ has not admired any pig', where, Geach says, he uses " "the man $a$ " as proxy 
for a term that is a name of and for a man'. In that case, since 'Socrates' is a name of and for a man, 'Socrates has not admired any pig' validly follows from the premise by itself; there is no need to add a premise stating that Socrates is or was a man.

The examples in this paper have not shown an inconsistency in Geach's system. That they are surprising examples I think shows us that we do not very readily know our way about logically, given Geach's style of restricted quantification together with his theory of relative identity.

Here I must mention one problem that has not been one of my concerns in this paper; it involves the use of schematic letters in displaying syllogistic patterns. Consider the sentence 'all men are mortal'. We have treated this sentence as having the form 'all $A$ are $B$ ' where ' $A$ ' and ' $B$ ' are in the same syntactic category. Certainly this schematization is problematic ('all mortal are men' does not even make good sense). ${ }^{3}$ But, in any case, we can recognize a common logical form in, say, the propositions: 'all men are mortal', 'all heralds are British', 'all dogs are plants'. How we adequately schematize this form will depend in part upon whether Geach's theories or some other logical theories are correct. But we do need some way to indicate what form of proposition or argument we are speaking about. To this end we have used the traditional schematizations because anyone should then know what form of proposition or argument we have in mind.

I believe that the general view among philosophers is that however one might have to tidy things up to get a theory of syllogisms in proper formal shape - any argument is valid if we would now (prior to tidying things up) be willing to informally treat it as instantiating a traditionally accepted syllogistic pattern (with the exception of cases where validity depends upon universal quantifiers having been given existential commitment). We now see that if we accept Geach's claims regarding relative identity and restricted quantifiers, we must reject this general view. With the examples in this paper we can see how radically Geach's theories would change what we count as a valid argument.

University of Pennsylvania

C JAMES CAIN 1985

\footnotetext{
${ }^{3}$ Note that in my examples the only terms I have used in subject position are substantival terms, and counterexamples for each syllogistic form can be provided with the subject terms being only substantival terms.
} 